\title{
CUSTOS DA UNIDADE DE HEMODIÁLISE DO HOSPITAL DAS CLÍNICAS DE PERNAMBUCO EM 2016
}

\author{
M. de F. FONSECA ${ }^{1}$ e S. A. VIDAL ${ }^{2}$ \\ ${ }^{1}$ Universidade Federal de Pernambuco, Centro de Ciências Sociais Aplicadas, Programa \\ de Gestão e Economia da Saúde - PPGGES \\ ${ }^{2}$ Universidade Federal de Pernambuco, Centro de Ciências Sociais Aplicadas, Programa \\ de Gestão e Economia da Saúde - PPGGES \\ E-mail: pesquisa.artigo10@gmail.com
}

RESUMO - A doença renal crônica (DRC) é um problema de saúde pública. No Brasil, o quantitativo de pacientes dependentes de terapia renal substitutiva (TRS) aumenta a cada ano, gerando preocupações crescentes em relação à sua eficiência e seus custos, visto abranger procedimentos de alta complexidade e existir carência desses dados. Objetivo: Analisar os custos e determinar o ponto de equilibrio da unidade de hemodiálise do Hospital das Clínicas de Pernambuco em 2016. Método: Pesquisa de descrição retrospectiva de custo de caso único baseada no método de custeio variável. Resultados e discussão: A unidade de hemodiálise (HD) realizou um total de 9.952 sessões de HD equivalente a 829,33 sessões/mês, com repasse do SUS mensal de R\$ 171.821,2. Do total dos custos, 90,57\% são fixos e 9,43\% são custos variáveis. Dos CF o gasto com pessoal é predominante e representa 77,16\% dos CT e 85,19\% dos CF. Dentre os CV os materiais de consumo somam 93,17\% desses custos. Entretanto, no montante do CT, esse item representa apenas $8,78 \%$. A margem de contribuição foi de $R \$ 134.537,69$ e o ponto de equilíbrio em relação à receita total (RT) igual a $R \$ 457.352,79$ gerando um déficit mensal de $R \$ 223.615,28$. Conclusão: A unidade de $H D$ opera em níveis inferiores ao ponto de equilibrio, ou seja, a RT obtida através das sessões de hd realizadas, com base na tabela SUS, não é suficiente para cobrir o CT, nem a MC é suficiente para cobrir os custos e despesas da instituição e o resultado apurado é negativo.

Palavras-chave: Terapia Renal Substitutiva, Hemodiálise, Métodos de custeio, Custeio variável.

ABSTRACT - Chronic kidney disease (CKD) is a public health problem. In Brazil, the number of patients dependent on renal replacement therapy (SRT) increases each year, generating increasing concerns regarding its efficiency and costs, since it covers procedures of high complexity and there is a lack of data. Objective: To analyze the costs and determine the break-even point of the hemodialysis unit of the Hospital das Clinicas of Pernambuco in 2016. Method: Retrospective description of single-case cost based on the variable cost method. Results and discussion: The hemodialysis unit (HD) performed a total of 9,952 HD sessions equivalent to 829.33 sessions / month, with a monthly passthrough of the SUS of $R \$ 171,821.2$. Of the total costs, $90.57 \%$ are fixed and $9.43 \%$ are variable costs. Of the $C F$, the expenditure with personnel is predominant and represents $77.16 \%$ of TC and $85.19 \%$ of CF. Among the CVs, consumer materials account for 
93.17\% of these costs. However, in the TC amount, this item represents only $8.78 \%$. The contribution margin was $R \$ 134,537.69$ and the break-even point in relation to total revenue (RT) was $R \$ 457,352.79$, generating a monthly deficit of $R \$ 223,615.28$. Conclusion: The HD unit operates at levels lower than the equilibrium point, that is, the $R T$ obtained through the hd sessions performed, based on the SUS table, is not enough to cover the CT, nor is MC sufficient to cover the costs and expenses of the institution and the result is negative.

Keywords: Renal replacement therapy, Hemodialysis, Costing methods, Variable costing.

\section{INTRODUÇÃO}

A doença renal crônica (DRC) consiste em lesão renal e perda progressiva e irreversível da função dos rins. Atualmente é considerada um problema de saúde pública em todo o mundo, sendo estimada prevalência variando entre 8 e $16 \%$. Apesar de cerca de $80 \%$ dos casos da doença ser observado em países desenvolvidos, é descrito um padrão de aumento na incidência da doença em países com diferentes características econômicas (MENEZES, 2015, p.368).

A DRC em seu estagio terminal é denominada de Insuficiência renal, a qual há necessidade de terapia renal substitutiva. Contudo, Apesar de fundamental para estes pacientes, a utilização da hemodiálise como terapia renal substitutiva implica em elevado custo econômico e social. (MENEZES, 2015)

Diante desta problemática, o objetivo do estudo foi analisar os custos e determinar o ponto de equilíbrio da unidade de hemodiálise do Hospital das Clínicas de Pernambuco em 2016. Estando este estudo justificado pela necessidade de se analisar os custos da unidade de hemodiálise, uma vez que esses procedimentos tendem a elevar os gastos para a instituição, refletindo diretamente na oferta desse tratamento (sessão de hemodiálise) aos pacientes e corroborando como subsídio para tomada de decisão dos gestores.

\section{MATERIAIS E MÉTODOS}

É uma pesquisa de descrição retrospectiva de custo de caso único realizada na unidade de hemodiálise do Hospital das Clínicas de Pernambuco no período de 2016, baseada no custeio variável. Sendo adotado o método de estudo de caso, por se tratar de uma pesquisa de caráter de profundidade e detalhamento (VERGARA, 2010), buscando uma investigação detalhada dos processos desenvolvidos na unidade de análise.

Optou-se pelo uso do método de custeio variável, pois através deste método foi possível estudar os custos em função do volume de sessões de hemodiálise realizadas em 2016. Possibilitando analisar se a quantidade de procedimentos realizados pela unidade dialítica é suficiente para cobrir seus custos e despesas fixas e manter o equilíbrio financeiro da instituição. Contudo, o sistema de custeio variável fundamenta-se na separação dos gastos variáveis e fixos. Trata-se de um método que faz a análise dos gastos variáveis (diretos e indiretos, custos ou despesas) e sua confrontação com as receitas (BRASIL, 2013). Os dados foram coletados através do sistema de informação hospitalar e da análise documental existente no setor de divisão de custos. Em seguida, Criou-se uma 
planilha de Excel, onde os dados referentes aos investimentos e despesas foram compilados. Posteriormente foi realizada a identificação dos custos diretos e indiretos do centro hemodialítico do HC e após, foram classificados em custos fixos e custos variáveis.

\section{RESULTADOS E DISCUSSÃO}

No ano de 2016 a unidade de hemodiálise (HD) do hospital das clínicas de Pernambuco realizou um total de 9.952 sessões de HD, equivalente a uma média mensal de 829,33 sessões/mês, com repasse do SUS mensal de R \$171.821,2. Na tabela abaixo se observa a quantidade de sessões realizadas, por tipo de sessão, o total anual de repasses realizado pelo SUS e o valor unitário fixo pago por cada tipo de tratamento hemodialítico.

Tabela 1. Quantidade de sessões de HD ofertada em 2016 e repasse do SUS em 2016, com valor fixo unitário por tipo de sessão, no Hospital das Clínicas, Recife, 2017.

\begin{tabular}{|c|c|c|c|c|}
\hline TIPO DE SESSÕES & $\begin{array}{l}\text { QUANT. ANUAL } \\
\text { DE SESSÕES }\end{array}$ & $\%$ & $\begin{array}{l}\text { VALOR ANUAL } \\
\text { (R\$) }\end{array}$ & $\begin{array}{c}\text { VALOR } \\
\text { UNITÁRIO (R\$) }\end{array}$ \\
\hline $\begin{array}{l}\text { HEMODIALISE (MAXIMO } 3 \\
\text { SESSOES POR SEMANA) }\end{array}$ & 7.886 & 79,2 & $1.531 .461,2$ & \\
\hline $\begin{array}{l}\text { HEMODIALISE (MAXIMO } 1 \\
\text { SESSOES POR SEMANA) }\end{array}$ & 252 & 2,6 & $48.938,4$ & 194,20 \\
\hline $\begin{array}{lr}\text { HEMODIALISE } & \text { EM } \\
\text { PACIENTE } & \text { COM }\end{array}$ & 176 & 1,8 & $46.712,16$ & \\
\hline SOROLOGIA POSITIVA & & 16,4 & & 265,41 \\
\hline $\begin{array}{l}\text { HEMODIALISE } \\
\text { PACIENTES } \\
\text { AGUDOS }\end{array}$ & 1.635 & & $433.945,35$ & \\
\hline HEMODIALISE CONTINUA & 3 & 0,0 & 796,23 & \\
\hline TOTAL & 9.952 & 100 & 2.061.853,34 & \\
\hline
\end{tabular}

Fonte: Mastertools, 2017.

Observa-se que o tipo de sessão predominante no centro dialítico da unidade de análise foi a hemodiálise com no máximo três sessões por semana $(79,2 \%)$, sendo justificado porque esse tipo de tratamento é destinado aos pacientes crônicos que realizam a terapia dialítica três vezes por semana durante quatro horas.

Conforme evidenciado no gráfico 1, os custos foram classificados quanto ao volume de produção em custos fixos (CF) ou variáveis (CV). Os CF representam 90,57\% dos custos totais da unidade de hemodiálise, enquanto que os CV representam 9,43\%. 
Gráfico 1 - Porcentagens dos Custos Fixos e Variáveis da Hemodiálise.

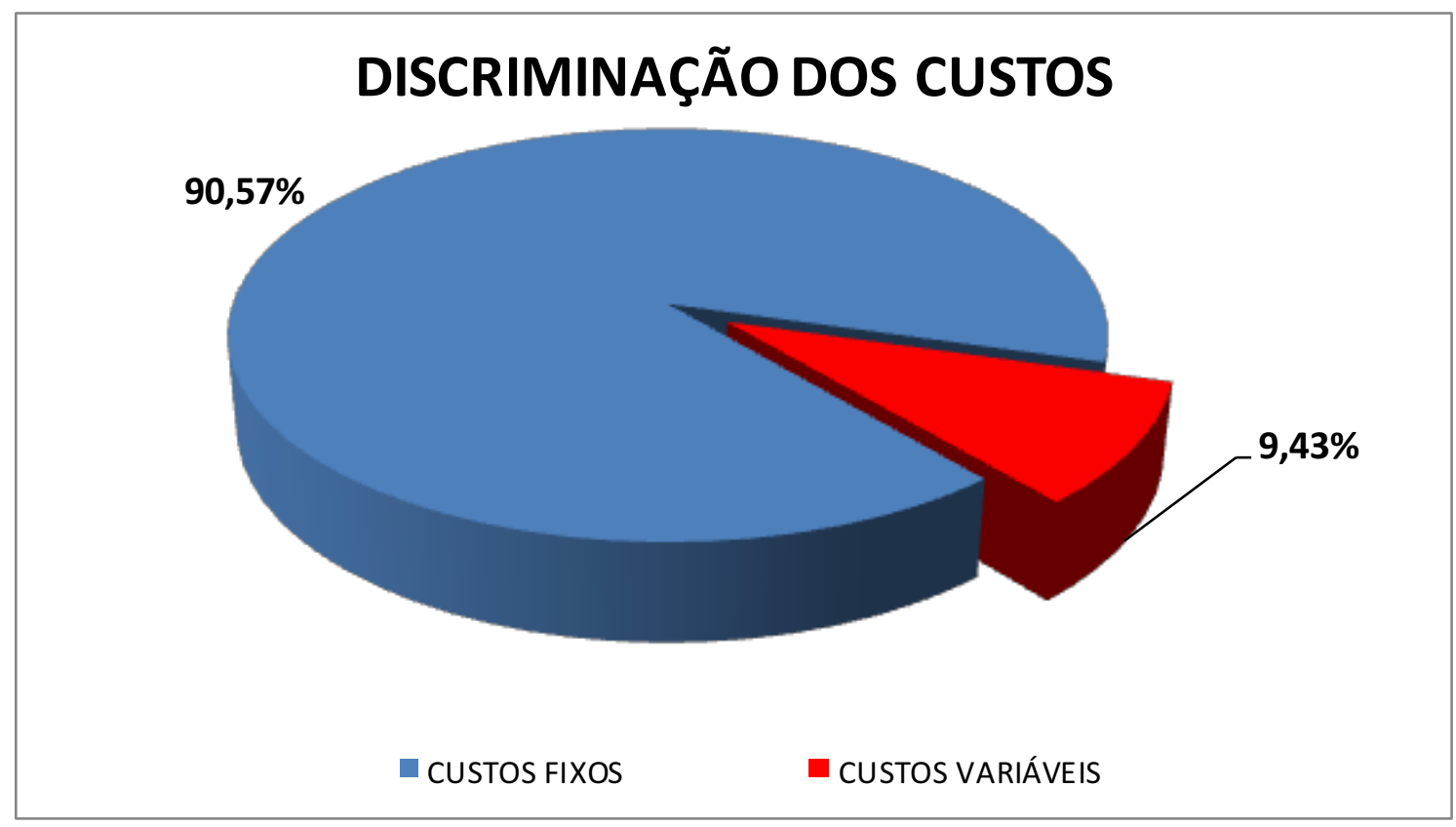

Fonte: Elaborado pela autora, 2017.

Dentre os custos fixos, evidencia-se que o gasto com a folha de pagamento dos recursos humanos representa o maior custo da unidade de hemodiálise (R $\$ 305.113,34$ ). Os gastos com esse grupo de custos representam $77,16 \%$ dos custos totais e $85,19 \%$ dos custos fixos do centro dialítico. Conforme evidenciado na tabela 2. Este achado corrobora com o estudo de Hsiao (2015), cujo gasto com recursos humanos correspondeu a 65,04\%.

Tabela 2 - Custos Fixos da unidade de hemodiálise do Hospital das Clínicas.

\begin{tabular}{c|c|c|c}
\hline \multicolumn{1}{c|}{ CUSTOS FIXOS } & TOTAL ANUAL R\$ & MÉDIA MENSAL R\$ & $\%$ \\
\hline $\begin{array}{l}\text { INVESTIMENTO/DEPRE } \\
\text { CIAÇAO }\end{array}$ & $30.036,72$ & $2.503,07$ & $0,70 \%$ \\
\hline RECURSOS HUMANOS & $3.661 .359,76$ & $305.113,34$ & $85,19 \%$ \\
\hline CONTRATOS & $606.438,72$ & $50.536,56$ & $14,11 \%$ \\
\hline \multicolumn{1}{c|}{ TOTAL } & $\mathbf{4 . 2 9 7 . 8 3 5 , 2 0}$ & $\mathbf{3 5 8 . 1 5 2 , 9 7}$ & $\mathbf{1 0 0 \%}$ \\
\hline
\end{tabular}

Fonte: Elaborado pela autora, 2017.

Vale ressaltar, que os salários dos recursos humanos foram depreciados e contabilizados de acordo com o tempo dedicado exclusivamente a unidade de hemodiálise, ou seja, por hora trabalhada.

Por outro lado, os custos variáveis corresponderam ao somatório dos materiais de consumo usados mensalmente nos procedimentos, acrescidos dos gastos com manutenção predial, energia elétrica e telefone. Conforme apresentado na tabela 3 . 
Tabela 3 - Custos Variáveis da unidade de hemodiálise do Hospital das Clínicas.

\begin{tabular}{c|l|c|c|c}
\hline \multicolumn{2}{c|}{ CUSTO VARIÁVEL } & $\begin{array}{c}\text { VALOR } \\
\text { TOTAL (R\$) }\end{array}$ & $\begin{array}{c}\text { MÉDIA } \\
\text { MENSAL R\$ }\end{array}$ & $\%$ \\
\hline \multirow{3}{*}{} & & & & $19,40 \%$ \\
& MATERIAL MÉDICO HOSPITALAR & $86.761,60$ & $7.230,14$ & \\
\cline { 2 - 5 } & MEDICAMENTOS & $289.794,93$ & $24.149,58$ & $64,77 \%$ \\
\cline { 2 - 5 } & MATERIAL DE EXPEDIENTE & 752,86 & 62,74 & $0,17 \%$ \\
\cline { 2 - 5 } & MATERIAL QUÍMICO & $38.922,89$ & $3.243,57$ & $8,70 \%$ \\
\cline { 2 - 5 } & MATERIAL DE COPA E COZINHA & 548,91 & 45,74 & $0,12 \%$ \\
\cline { 2 - 5 } & CONSUMO GERAL & 33.91 & 2,82 & $0,01 \%$ \\
\hline \multirow{3}{*}{} & MANUTENÇÃO PREDIAL & $1.021,44$ & 85,12 & $0,23 \%$ \\
\hline & ENERGIA ELETRICA & $29.187,75$ & $2.432,32$ & $6,52 \%$ \\
\hline & TELEFONE & 377,7 & 31,48 & $0,08 \%$ \\
\hline & & $\mathbf{4 4 7 . 3 6 8 , 0 8}$ & $\mathbf{3 7 . 2 8 3 , 5 1}$ & $\mathbf{1 0 0 \%}$ \\
\hline
\end{tabular}

Fonte: Elaborado pela autora, 2017.

Dentre os custos variáveis a maior parcela recai sobre os materiais de consumo, que somam $93,17 \%$ desses custos. Entretanto, no montante dos custos totais, o material de consumo representa apenas 8,78\%.

\section{Cálculo pelo método de Custeio Variável}

Para obtenção da receita total (RT) da unidade de hemodiálise multiplicou-se a média mensal de sessões de HD pelos respectivos valores monetários em Reais (R\$) das sessões, com base na tabela de procedimentos do SUS. Neste cenário, a RT mensal do centro dialítico é R\$ $171.821,2$, conforme demonstrado no quadro 1 abaixo.

Quadro 1 - Receita Total. Unidade de Hemodiálise do Hospital das Clínicas de Pernambuco em 2016.

\begin{tabular}{|l|c|c|c|}
\hline \multicolumn{5}{|c|}{ RECEITA TOTAL } \\
\hline TIPO DE SESSÃO & $\begin{array}{c}\text { MÉDIA MENSAL } \\
\text { DE SESSÃO (A) }\end{array}$ & $\begin{array}{c}\text { VALOR } \\
\text { TABELA SUS } \\
\text { (B) }\end{array}$ & $\begin{array}{c}\text { RECEITA (C) } \\
\text { (C=A x B) }\end{array}$ \\
\hline HD Máximo 3 sessões/semana & 657,16 & 194,20 & $127.621,5$ \\
\hline HD Máximo 1 sessão/semana & 21 & 194,20 & $4.078,2$ \\
\hline HD em pacientes renais agudos & 136,25 & 265,41 & $36.163,1$ \\
\hline HD paciente c sorologia + & 14,66 & 265,41 & $3.891,0$ \\
\hline HD Contínua & 0,25 & 265,41 & 67,4 \\
\hline \multicolumn{7}{|r|}{} & TOTAL & R\$ 171.821,2 \\
\hline
\end{tabular}

Fonte: Elaborado pela autora. 
Na tabela 4 apresenta-se o cálculo da margem de contribuição (MC) e do ponto de equilíbrio $(\mathrm{PEq})$ da unidade de hemodiálise, com base na receita total. A margem de contribuição encontrada no valor monetário de $\mathrm{R} \$ 134.537,69$ indica que a receita total não cobre os custos fixos do centro hemodialítico ( $\mathrm{R}$ \$ 358.152,97), ou seja, a instituição não atinge um nível positivo de contribuição marginal, gerando um déficit mensal de $\mathrm{R} \$$ $223.615,28$.

O percentual dos CV em relação a RT foi de $21,69 \%$ e a diferença entre o percentual dos $\mathrm{CF}$ em relação $\mathrm{CV}$ foi de $78,31 \%$. Logo, enfatiza-se que um elevado CF contribui para o aumento do Ponto de Equilíbrio e ocasiona riscos para a continuidade da atividade operacional.

A análise do PEq em relação a receita total aponta um valor de $\mathrm{R} \$ 457.352,79$. Este valor indica que para a terapia hemodialítica, a instituição teria que aumentar sua receita em $62,43 \%(100 \%-37,57 \%)$ para suprir seus custos totais $(\mathrm{CF}+\mathrm{CV})$ e alcançar o equilíbrio financeiro. Este dado demonstra que o nível de cobertura da receita recebida pelas sessões de hemodiálise realizadas no centro dialítico do hospital das clínicas de Pernambuco em 2016, se encontra expressivamente abaixo do valor monetário que é necessário para cobrir integralmente seus custos e para otimizar a operacionalização do serviço.

Tabela 4- MC, PEq e Déficit de receita/mensal. Unidade de Hemodiálise, 2016.

\begin{tabular}{|c|c|c|}
\hline & DESCRIÇÃO & VALOR MENSAL RS \\
\hline (A) & Receita Total (RT) & $\mathrm{R} \$ 171.821,2$ \\
\hline (B) & Total dos custos fixos (CF) & $\mathrm{R} \$ 358.152,97$ \\
\hline (C) & Total dos custos variáveis (CV) & $\mathrm{R} \$ 37.283,51$ \\
\hline$D=(A-C)$ & MARGEM DE CONTRIBUIÇÃO & R\$ $134.537,69$ \\
\hline$E=(C / A)$ & \% CV em relação à $\mathrm{RT}$ & $21,69 \%$ \\
\hline$F=(100 \%-E)$ & \% Diferença de CF em relação aos CV & $78,31 \%$ \\
\hline$G=(B / F)$ & $\begin{array}{llcl}\text { PONTO } & \text { DE } & \text { EQUILÍBRIO } & \text { EM } \\
\text { RELAÇÃO À RECEITA } & \end{array}$ & R\$ 457.352,79 \\
\hline$H=(A / G)$ & \% de execução da capacidade de equilíbrio & $37,57 \%$ \\
\hline$I=(D-B)$ & DÉFICIT DA RECEITA & - R\$ 223.615,28 \\
\hline
\end{tabular}

Fonte: Elaborado pela autora.

É possível inferir, que a unidade de hemodiálise opera em níveis inferiores ao ponto de equilíbrio, ou seja, a RT obtida através das sessões de hemodiálise realizadas, com base na tabela SUS, não é suficiente para cobrir o CT. A MC não é suficiente para cobrir os custos e despesas da instituição e o resultado apurado é negativo.

$\mathrm{Na}$ tabela 5 evidencia-se a MC utilizando como RT o valor do PEq encontrado. Verifica-se que este valor corresponde exatamente à diferença entre o valor da Receita Total e os custos variáveis, ou seja, o valor necessário para cobrir os custos fixos e manter o equilíbrio financeiro. Observa-se que há um aumento dos custos variáveis para $\mathrm{R} \$$ 99.199,82, já que estes custos se modificam conforme variação da receita.

Tabela 5 - Evidenciação da MC dado PEq encontrado (Receita ideal).

\begin{tabular}{c|l|l}
\hline & DESCRIÇÃO & VALOR MENSAL RS \\
\hline (A) & Receita Total & $\mathrm{R} \$ 457.352,79$ \\
\hline (B) & Total dos custos fixos $(78,31 \%)$ & $\mathrm{R} \$ 358.152,97$ \\
\hline (C) & Total dos custos variáveis $(21,69 \%)$ & $\mathrm{R} \$ 99.199,82$ \\
\hline $\mathrm{D}=(\mathrm{A}-\mathrm{C})$ & MARGEM DE CONTRIBUIÇ $\tilde{\mathbf{A} O}$ & $\mathrm{R} \$ 358.152,97$ \\
\hline \multicolumn{2}{|l}{}
\end{tabular}


Portanto, com a receita ideal (PEq) o hospital cobriria seus custos fixos e atenderia a demanda de 829,33 sessões/mês. Contudo, com a receita real a instituição deveria ofertar em torno de 4.144 sessões de hemodiálise a menos, encontrada dividindose o custo variável da receita real $(\mathrm{R} \$ 186.331,77)$ pelo custo por sessão $(44,96)$. Sendo este custo por sessão a divisão dos custos variáveis (R $\$ 37.283,51)$ pelo total de sessões/ mês $(829,33)$.

\section{CONCLUSÃO}

A instituição já inicia suas atividades no prejuízo e que a receita atual não é suficiente para cobrir seus custos fixos, pois para cobri-los seria necessário um aumento da receita ou uma redução na quantidade ofertada de sessões de hemodiálise. Logo, a unidade hospitalar opera no prejuízo, pois o reembolso fixo para 829,33 sessões/mês repassado pelo Sistema Único de Saúde (SUS) é insuficiente, pois não cobre ao menos o custo fixo da unidade hospitalar.

Portanto, a unidade de hemodiálise opera em níveis inferiores ao ponto de equilíbrio (receita ideal), ou seja, a receita total obtida através das sessões de hemodiálise realizadas, com base na tabela SUS, não é suficiente para cobrir os custos totais, assim como, a margem de contribuição também não é suficiente para cobrir os custos e despesas da instituição e o resultado apurado é negativo, gerando um déficit financeiro na operacionalização do serviço.

Além disso, pressupõe-se que estes dados ainda estejam subestimados, pois não há registros das sessões de hemodiálises que foram realizadas em 2016 e não foram pagas pelo SUS, uma vez que este paga apenas por uma sessão extra por semana, além das três sessões realizadas normalmente. Logo, as sessões de hemodiálise que são ofertadas aqueles pacientes que realizam a terapia hemodialítica diariamente, de segunda a sábado, não serão reembolsadas. Terão que ser cobertas financeiramente pela instituição, ou seja, gerando um déficit financeiro ainda maior para a referida unidade, pois terão que arcar com as sessões realizadas não cobertas pelo SUS.

\section{REFERÊNCIAS}

BRASIL. Ministério da Saúde. Introdução à Gestão de Custos em Saúde / Ministério da Saúde, Organização Pan-Americana da Saúde. - Brasília : Editora do Ministério da Saúde, 2013. 148 p. : il. - (Série Gestão e Economia da Saúde ; v. 2).

HSIAO, J; LIMA, A.F.C. Custo direto da hemodiálise em unidade de terapia intensiva adulto. Cogitare Enferm. São Paulo, 2015 Out/dez; 20(4): 678-686.

MENEZES, Fabiana Gatti de et al. Overview of hemodialysis treatment funded by the Brazilian Unified Health System - An economic perspective. J. Bras. Nefrol., São Paulo, v. 37, n. 3, p. 367-378, Sept. 2015.

VEGARA, Sylvia Constant. Projetos e Relatórios de Pesquisa em Administração. 12. ed. São Paulo: Atlas, 2010. 